\title{
PROPOSTA DE ARQUITETURA DE UM PORTAL DE PROVAS SEGURO UTILIZANDO SDN COMO SOLUÇÃO DE GERENCIAMENTO DE REDES
}

\author{
João Paulo Pimentel, Georges Daniel Amvame Nze, Fábio Lúcio Lopes de Mendonça, \\ Robson de Oliveira Albuquerque, Vanessa Coelho Ribeiro e Rafael Timóteo de Sousa Júnior \\ Programa de Pós-Graduação Profissional em Engenharia Elétrica (PPEE), Departamento de Engenharia Elétrica, \\ Universidade de Brasília, Brasília, Brasil, Zipcode 70910-900
}

\begin{abstract}
RESUMO
Este artigo apresenta a proposta de um Portal Seguro de Provas com autenticação única utilizando SSO (Single Sign-On) e geração de token através de um código de barras bidimensional ( $Q R$ Code) gerado para a validação do usuário que deseja acessar o referido Portal com o intuito de realizar as suas avaliações previamente agendadas. Como solução de Gerenciamento de Redes, a proposta é de utilizar Redes definidas por software, ou seja, o SDN (Software Defined Networks).
\end{abstract}

\section{PALAVRAS-CHAVE}

Portal, SSO, QR Code, Token, SDN

\section{INTRODUÇÃ̃O}

A proposta deste artigo é de apresentar uma arquitetura de Portal de Provas seguro com autenticação SSO (Single Sign-On), utilizando QR Code (Quick Response Code) com geração de Token para os usuários do sistema e utilizando SDN (Software Defined Networking) como solução de gerenciamento de redes. Neste Portal o usuário terá a segurança de autenticação única e segura é feito justamente para os alunos irem realizar as suas avaliações de acordo com o seu agendamento no Portal que após a escolha da referida disciplina, data e horário será gerado um $Q R$ Code para ser validado e confirmado ao entrar no laboratório que irá realizar a referida avaliação.

A autenticação SSO que é para validar e confirmar que é você mesmo que está utilizando o sistema Web, tem a ideia de exigir uma mesma senha para todos os acessos de forma segura e transparente, evitando com isso, que tenha que anotar ou tenha que gerar uma senha para cada tipo de aplicativo que estiver utilizando, ou seja, como a autenticação SSO, o usuário digita apenas uma vez o login e a senha no primeiro acesso e depois vai abrindo os demais aplicativos sem a necessidade de digitar novamente a senha. Um bom exemplo desta autenticação é a utilização da autenticação do seu e-mail que validará que é você mesmo que está utilizando o sistema $W e b$, então deixaria você acessar o Portal para agendar a sua avaliação.

Outra validação de segurança do SSO seria o envio de código de validação para o seu número de celular ou e-mail para confirmar se é você mesmo que está utilizando o referido sistema Web, com isso, aumentaria a confiança sobre esta utilização.

O QR Code com Geração de Token neste Portal seria enviado para o e-mail do usuário cadastrado e seria utilizado no momento da entrada do aluno no destino da realização de sua avaliação, ou seja, no laboratório agendado. Ao apresentar o QR Code seria gerado um Token para ser utilizado no login no Portal na estação de trabalho agendada para o determinado login, ou seja, o Token somente será válido na máquina que foi liberada no momento do agendamento pelo aluno.

Este agendamento em máquinas específicas será realizado utilizando SDN como Solução de Gerenciamento de Redes e o OpenFlow como protocolo de comunicação, ou seja, um controlador SDN localizado na central de monitoramento com um plano definido fica responsável por gerenciar os switches da rede, liberando o acesso pelo Token informado somente naquela porta de rede que encontra a máquina que o aluno vai realizar a referida avaliação. 


\section{REFERENCIAMENTO TEÓRICO E ARQUITETURA PROPOSTA}

O gerenciamento baseado em SDN que será controlado por um elemento central, ficou conhecido por separar do controle da rede dos dispositivos de encaminhamento, tais como roteadores, switches, fazendo com esta lógica fosse definida por software de forma centralizada, provendo à rede através da utilização de aplicações customizadas para cada cenário definido (Mckeown et al., 2008) e estas aplicações implementam a inteligência da rede e proveem abstração dos detalhes técnicos dos equipamentos e dos protocolos.

O OpenFlow é a principal interface de código aberto entre SDN controladores e comutadores ou outros caminhos de dados. Possui suporte para diversos aplicativos usando protocolos de planos de dados com a classificação de pacotes sendo executadas usando tabelas de correspondência e operações de processamento de pacotes chamadas de ações ou instruções (Software-Defined Networking, 2016). O OpenFlow é um padrão definido pela Open Networking Foundation (ONF) para implementar SDN em equipamentos de rede e define a interface entre um controlador OpenFlow e um comutador OpenFlow, ou seja, ele permite que o OpenFlow Controller instrua o comutador OpenFlow sobre como lidar com pacotes de dados recebidos (Noviflow, 2020).

A autenticação SSO na Web, como exemplo do Login do Google ou Facebook, são baseados no protocolo OpenID Connect (Miculan, M., Urban, C, 2011). Esse protocolo permite que através da confiança capitam a autenticação do usuário aos provedores de identidade, ou seja, é através dele que é passada a identificação do usuário para a devida autenticação e validação nas aplicações Web. No SSO, um serviço da Web, nesse contexto chamado RP (Relying Party), delega autenticação para o chamado provedor de identidade (IdP). A tarefa de um IdP no SSO da Web é autenticar usuários e atestar suas identidades aos RPs. Alguns serviços que os usuários usam diariamente como provedores de $e$-mail ou redes sociais, servem como IdPs (R. Barnes, 2011).

Hoje, diversos usuários da Web utilizam suas contas do Facebook ou do Google para acessar sites de terceiros (Relying Party). Esse esquema de login único baseado na Web é ativado pelo OAuth 2.0, um protocolo de autorização de recursos da Web que foi adotado pelos principais provedores de serviços (Patterson, 2014). O QR Code (Quick Response Code) que é utilizado para resposta rápida (Pillai, A. E., Prakash, D., Al-Marhoobi, N. A., \& Shrivastava, M., 2017) tem a vantagem nesta proposta do Portal, pois vem da facilidade em realizar a leitura durante a entrada no laboratório para realizar a devida prova.

A arquitetura proposta neste trabalho será de pôr autenticação SSO, geração de um Token através de um QR Code gerado para a autenticação e validação de um determinado usuário (o aluno). Na sequência o aluno irá acessar o Portal a fim de realizar o agendamento da sua devida avaliação. A ideia central do do sistema é a autenticação/validação dos usuários através de SSO, que na sequência terá a opção do aluno escolher qual avaliação irá fazer e com isso será gerado um $Q R$ Code no qual o aluno deverá apresenta-lo no laboratório que será realizada a devida avaliação. Após apresentar o $Q R$ Code no laboratório previamente agendado para a devida avaliação, este gerenciamento será resolvido com a utilização de SDN, o módulo de autenticação/validação deste Portal irá gerar um Token válido somente para a avaliação solicitada na máquina liberada. Com a utilização da matrícula e o Token gerado pelo Portal Web, o aluno irá logar no referido Portal de Provas e realizar a devida avaliação.

Nesta arquitetura proposta, para ficar mais seguro, o Token enviado para o aluno que será validado somente em uma máquina específica do laboratório, terá um prazo para expirar de 20 minutos, ou seja, se o aluno não realizar o login no referido Portal para realizar a sua avaliação nestes 20 minutos, ele terá que solicitar novamente um novo código que poderá mudar até de estação de trabalho para a realização da referida avaliação.

Sobre o gerenciamento via SDN, este módulo deverá ter uma configuração para enviar automaticamente as requisições dos alunos para os destinos (máquinas nos laboratórios) através do plano de controle de um controlador SDN, utilizando como protocolo de comunicação o OpenFlow. Este controlador SDN enviará as informações para os switches que comunicarão com as máquinas definidas para a realização de cada avaliação e na hora da realização da Prova, ao digitar a matrícula e token recebido após a validação do agendamento, serão confirmados na tabela do plano de controle SDN utilizando uma Interface para comunicar com o Portal.

Para o desenvolvimento deste Portal, será utilizada o Framework Flutter que é um kit de ferramentas de interface do usuário desenvolvido pelo Google para criar aplicativos que são compilados de forma nativa para dispositivos móveis, Web e desktop a partir de uma única base de código fonte (Flutter, 2020). A linguagem de programação utilizada neste Portal, será a linguagem Dart, que foi desenhada pelo Google para aplicações Web modernas e capacitadas para ambientes de alta performance (Dart, 2020). A base de dados para este Portal será o Firebase Realtime Database, que além de base de dados, é uma plataforma de 
desenvolvimento de fácil integração na Web. Os dados são armazenados como objetos JSON (JavaScript Object Notation) na nuvem, ou seja, ao contrário de um banco de dados SQL, não há tabelas e nem registros, mas sim uma árvore JSON (Firebase, 2020).

Este Portal terá três tipos de perfis de acessos, um de aluno que somente agenda e realiza a sua avaliação, outro de Professor para alimentar o Banco de Questões do referido Portal e liberação das provas e o de Coordenador que revisa e valida questões incluídas pelos Professores. A partir da validação das questões pelo Coordenador, estas questões serão liberadas para os Professores montarem suas provas e liberarem para serem realizadas pelos alunos.

\section{APRESENTAÇÃO DOS RESULTADOS INICIAIS}

Os resultados iniciais deste Portal, de acordo com a arquitetura proposta de desenvolvimento, apresentam uma tela de login na Figura 1 aonde o usuário tem a opção de realizar o login se já tiver cadastrado, ou cadastrar um login de sua preferência, ou simplesmente continuar utilizando a sua conta do Facebook ou da Google.

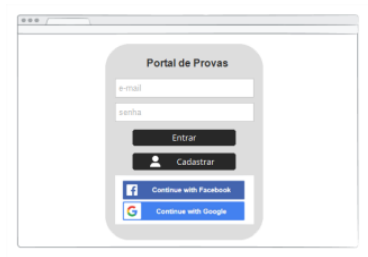

Figura 1. Tela de Login

Já a tela de agendamento de Provas, ilustrada na Figura 2, demostra como será este agendamento do usuário, aonde após logado serão apresentadas as informações do usuário logado, tais como nome, matrícula, curso que está matriculado. Nesta tela o usuário seleciona a disciplina que ele quer realizar o agendamento da Prova, nesta lista somente vão aparecer as disciplinas que o usuário está matriculado, pois este Portal deverá receber estas informações do Portal de Matrículas, na sequência escolhe uma data no calendário e automaticamente irá aparecer uma lista de horários disponíveis para o referido agendamento, escolhido o horário e clicado no botão agendar, irá gerar um $Q R$ Code para apresentação no dia da Prova no Laboratório agendado.

Com o $Q R$ Code gerado o usuário vai apresentá-lo no laboratório agendado, ao apresentar o referido $Q R$ Code ele receberá um Token no e-mail cadastrado e/ou número de celular cadastrado com validade de 20 minutos para realizar o login no Portal na máquina agendada juntamente com sua matrícula. A Figura 3 ilustra o modelo de mensagem que poderá chegar por e-mail ou SMS (Short Message Service).

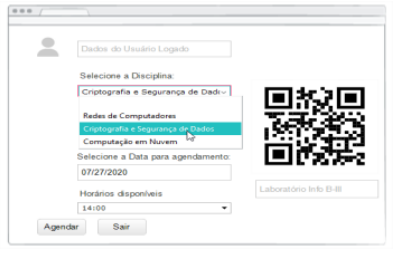

Figura 2. Tela de Agendamento

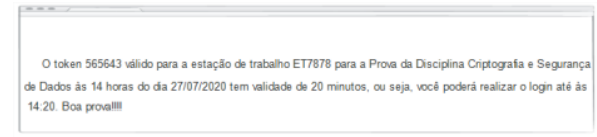

Figura 3. Tela do Token enviado ao usuário

Com o Token o usuário irá efetuar o login no Portal através do módulo de realização de Provas. 


\section{CONCLUSÃO E TRABALHOS FUTUROS}

Este artigo propôs uma arquitetura para um Portal de Provas Seguro para alunos realizarem os seus agendamentos das provas com segurança utilizando SSO para autenticação no Portal, QR Code para gerar um Token que será validado por um gerenciamento de Redes por controle SDN, com isso, garante que o aluno estará realizando a avaliação no laboratório que foi definido pelo gerenciamento SDN.

Os desafios para este Portal de Prova só estão começando, pois a integração deste Portal com a base de dados de um Portal de Matrícula deverá ser bem definida, a comunicação do Portal com o SDN com envio desta mensagem com o Token válido é uma inovação que garante que esta proposta seja aceita e utilizada no desenvolvimento de vários Portais de Provas em várias instituições de ensino.

Para trabalhos futuros fica a ideia de desenvolver um aplicativo mobile para comunicar com este Portal e os alunos além de registrarem suas presenças com o $Q R$ Code gerado para a aula, validando este $Q R$ Code ao entrar no laboratório ou sala de aula, como também realizar os agendamentos das avaliações e até quem sabe realizar as avaliações no aplicativo mobile, pois é a tendência de quase todas as aplicações Web, mas sempre lembrando de implementar uma segurança bem definida.

\section{AGRADECIMENTOS}

Os autores agradecem o apoio do Programa de Pós-Graduação Profissional em Engenharia Elétrica (PPEE) da UnB Agências brasileiras de pesquisa, desenvolvimento e inovação CNPq e CAPES, bem como o suporte do Laboratório LATITUDE/UnB a a cooperação o Gabinete de Segurança Institucional da Presidência da República / CEPESC (TED 002/2017).

\section{REFERÊNCIAS}

Dart. Disponível em: <https://dart.dev/>. Acessado em 10 jul. 2020.

Firebase. Disponível em: <https://firebase.google.com/docs/database/Web/structure-data?hl=pt-br>.Acessado em: 12 jul. 2020.

Flutter. Disponível em: <https://flutter.dev/>. Acessado em 10 jul. 2020.

Mckeown, N. et al. OpenFlow: enabling innovation in campus networks. SIGCOMM Comput. Commun. Rev., ACM, New York, NY, USA, v. 38, n. 2, p. 69-74, 2008.

Miculan, M., Urban, C.: Formal analysis of Facebook Connect Single Sign-On authentication protocol. In: Proc. SofSem 2011, OKAT, pp. 99-116 (2011)

Noviflow. The OpenFlow Protocol. Disponível em: <https://noviflow.com/the-basics-of-sdn-and-the-openflow-networkarchitecture/>. Acessado em: 11 jun. 2020.

Patterson, P. Digging deeper into OAuth 2.0 on force.com (2014). Disponível em <https://developer.salesforce.com/page/Digging_Deeper_into_OAuth_2.0_on_Force.com>. Acessado em 12 jun. 2020.

Pillai, A. E., Prakash, D., Al-Marhoobi, N. A., \& Shrivastava, M. (2017). Application of QR codes in tourism industry: A review of literature. International Journal of Computer Technology \& Applications, 8(6), 678-687.

R. Barnes. Use Cases and Requirements for DNS-Based Authentication of Named Entities (DANE). RFC 6394 (Informational). RFC. Fremont, CA, USA: RFC Editor, Oct. 2011. DOI: 10.17487/RFC6394. URL: https://www.rfceditor.org/rfc/rfc6394.txt.

Software-Defined Networking: ONF SDN Evolution. Open Networking Foundation, p. 8, 2016. Disponível em: <http://www.opennetworking.org/wp-content/uploads/2013/05/TR-535_ONF_SDN_Evolution.pdf>. Acessado em: 12 jun. 2020 